\title{
Periodontal treatment could improve glycaemic control in diabetic patients
}

\author{
Abstracted from \\ Darré L, Vergnes JN, Gourdy P, Sixou M. \\ Efficacy of periodontal treatment on glycaemic control in diabetic patients: a meta-analysis \\ of interventional studies. Diabetes Metab 2008; 34: 497-506 \\ Address for correspondence: Dr. M. Sixou, Dental Department of Epidemiology, Toulouse Dental Hospital, \\ 3, chemin des Maraîchers, 31062 Toulouse, France. E-mail: sixou@cict.fr
}

\section{Question: Does periodontal treatment have an effect on glycaemic control in diabetic patients?}

Data Sources Medline, Pascal, Embase, LILACS, the Cochrane Library, International Association of Dental Research Abstracts and Current Contents databases were searched and, in addition, a manual search was made of the bibliography of all pertinent articles. Unpublished articles were researched by contacting authors. No restrictions on language or type of article were applied in the literature search.

Study selection Two investigators reviewed the full texts independently. To be included in the meta-analysis, studies had to be original interventional studies, conducted on a human population in which subjects had both diabetes and periodontitis, and provided a numerical value for HbA1c (glycosylated haemoglobin level). Review articles and meta-analyses were excluded and only the most recent reference of a multiple publication was included.

Data extraction and synthesis The two reviewers independently extracted data using a standardised data-extraction grid. Data from the controlled studies were combined to estimate the pooled differential variation of $\mathrm{HbA} 1 \mathrm{c}$ between control and treatment groups, using the inverse-variance method. Both fixed and random-effects models were used to calculate the overall standardised mean difference (SMD). Statistical homogeneity across the studies was tested by Cochran's Q test. Potential publication bias was tested using the rank-correlation test for funnel-plot asymmetry.

Results Twenty-five studies, involving 976 subjects in total, were included in the present systematic review. Of these, nine studies, comprising 485 patients, were controlled trials and were included in the meta-analysis. The SMD in $\mathrm{HbA} 1 \mathrm{c}$ with the treatment of periodontal disease was 0.46 [95\% confidence interval $(\mathrm{CI})$, $0.11-0.82]$. These findings suggest that periodontal treatment could lead to a significant $0.79 \%(95 \% \mathrm{Cl}, 0.19-1.40)$ reduction in HbA1c level.

Conclusions The present meta-analysis represents the best information available to date that addresses this issue, and suggests that periodontal treatment could improve glycaemic control. Nevertheless, these results should be viewed with caution because of a lack of robustness and deficiencies in the design of some of the studies included. A randomised controlled trial (RCT) with sufficient statistical power would help to confirm the results of this meta-analysis.

\section{Commentary}

Evidence has steadily accumulated over the past 2 decades indicating, importantly, that oral conditions are related to systemic health and well-being. Biologically plausible mechanisms have been proposed to explain how oral infection and inflammation may be risk factors for several systemic health outcomes. There has been a relative paucity of experimental research in humans, however, to test whether or not treatment of oral disease leads to improved systemic health. To date, most of the evidence on oral-systemic relationships has come from observational studies which can only be viewed, at best, as 'hypothesis-generating' and not as 'hypothesis-testing.' Even the best epidemiological studies on the role that oral infection and inflammation may play as risk factors for various systemic outcomes are fraught with limitations. ${ }^{1}$ It remains to be conclusively demonstrated whether a causal relationship exists between oral conditions and systemic health, and whether oral conditions are modifiable risk factors.

Perhaps the most effort, and most progress, has been made in understanding the effects of periodontal disease on glycaemic control in diabetics. Thus, this work, by Darré et al., is timely and an important contribution in the field of oral-systemic links. It is a superbly conducted systematic review and meta-analysis of the question, "Does periodontal treatment have an effect on glycaemic control in diabetic patients?" It goes beyond an earlier review, that of Janket and colleagues ${ }^{2}$ in 2005, and encompasses published and unpublished studies through to 2007. Notably, multiple databases were searched without restrictions on language or type of article.

This systematic review identified a total of nine studies (485 patients in all) that were controlled trials and could be included in the meta-analysis. Although their meta-analysis indicated that periodontal treatment was effective in reducing $\mathrm{HbA1c}$ levels (eg, SMD in HbA1c was 0.46 ; 95\% CI, 0.11-0.82), the authors themselves express caution regarding the statistically significant, and clinically significant, finding. After conducting a sensitivity analysis, they discovered that exclusion of a single study, a non$\mathrm{RCT},{ }^{3}$ decreased the overall SMD to a nonsignificant value of 0.27 (95\% CI, -0.01-0.60). Thus, the most appropriate conclusion that can be drawn from this analysis is that we still lack conclusive evidence to recommend periodontal treatment as an effective means by which to reduce HbA1c levels in adult diabetics. Darré and coworkers note that a RCT with sufficient statistical power would be an important next step, and their post-hoc calculation showed that a minimum sample size of 150 participants would be necessary for such future RCT. 
Fortunately, such a study will soon be under way. The US National Institutes of Health has funded a large-scale, multicentre, definitive RCT on the effects of periodontal scaling and root planing in subjects who have type 2 diabetes and untreated, moderate to advanced, chronic periodontitis [see the URL crisp.cit.nih.gov/crisp/CRISP_LIB. getdoc?textkey=7438507\&p_grant_num=1U01DE01890201\&p_quer $\mathrm{y}=($ diabetes)\&ticket=86988031\&p_audit_session_id=402075696\&p_ audit_score=8\&p_audit_numfound=1\&p_keywords=diabetes]. The study will compare the 6-month change in HbA1c in 300 subjects randomly assigned to receive scaling and root planing (treatment group) with 300 subjects assigned to delayed treatment (control group). The proposed sample size has $90 \%$ power to detect a clinically relevant difference of $0.6 \%$ in $\mathrm{HbA1c}$ reduction within 6 months between the treatment and control groups. The trial is expected to be launched in 2009, but it is not likely to be completed until 2013 because of an estimated 30-month rolling enrolment period for the RCT. (NB. A Cochrane systematic review protocol on this question has been previously registered. $)^{4}$

Until definitive RCT results are available, it remains prudent for clinicians to continue to recommend periodontal treatment for diabetics for the primary purpose of improving their periodontal health. Whether such treatment can also improve their diabetic health status remains an open question.

Raul Garcia

Department of Health Policy and Health Services Research, and the Northeast Center for Research to Evaluate and Eliminate Dental Disparities, Boston University Goldman School of Dental Medicine, Boston, Massachusetts, USA

1. Dietrich T, Garcia RI. Associations between periodontal disease and systemic disease: evaluating strength of the evidence. J Periodontol 2005; 76: 2175-2184.

2. Janket S, Wightman A, Baird AE, Van Dyke TE, Jones JA. Does periodontal treatment improve glycemic control in diabetic patients? A meta-analysis of intervention studies. J Dent Res 2005; 84: 1154-1159.

3. Stewart J, Wager KA, Friedlander AH, Zadeh $\mathrm{HH}$. The effect of periodontal treatment on glycemic control in patients with type 2 diabetes mellitus. J Clin Periodontol 2001; 28: $306-310$.

4. Simpson T, Needleman I, Wild SH, Moles DR, Mills EJ. Treatment of periodontal disease for glycaemic control in people with diabetes. Cochrane Database Syst Rev 2004; issue 2.

Evidence-Based Dentistry (2009) 10, 20-21. doi:10.1038/sj.ebd.6400633 


\title{
Periodontal disease is a risk marker for coronary heart disease?
}

\author{
Abstracted from \\ Humphrey LL, Fu R, Buckley DI, Freeman M, Helfand M. \\ Periodontal disease and coronary heart disease incidence: a systematic review and meta-analysis. \\ J Gen Intern Med 2008; 23: 2079-2086 \\ Address for correspondence: Linda L Humphrey, Oregon Health and Science University, Mailcode BICC, \\ 3181 SW Sam Jackson Park Road, Portland OR 97239-3098, USA. E-mail: humphrey@ohsu.edu
}

\section{Question: Is periodontal disease an independent novel risk factor for incident coronary heart disease?}

Data Sources Relevant studies were identified using Medline and bibliographies of reviews, editorials, book chapters and letters discussing the relationship between periodontal disease and coronary heart disease (CHD).

Study selection Studies were assessed for inclusion by two reviewers. Prospective studies with cohort or nested case-control design with CHD or cardiovascular disease (CVD) as an outcome were included. Study quality was rated.

Data extraction and synthesis Data were abstracted by one reviewer and reviewed for accuracy by another author: any discrepancies were adjudicated by a third author. Meta-analysis was conducted to evaluate heterogeneity and publication bias.

Results Seven studies were included, some of which found that periodontal disease was independently associated with increased risk of CHD. Summary relative risk estimates for different categories of periodontal disease (including periodontitis, tooth loss, gingivitis and bone loss) ranged from 1.24 [95\% confidence interval $(\mathrm{Cl}), 1.01-1.51$ ] to $1.34(95 \% \mathrm{Cl}, 1.10-1.63)$. Risk estimates were similar in subgroup analyses by gender, outcome, study quality and method of periodontal disease assessment.

Conclusions Periodontal disease is a risk factor or marker for CHD that is independent of traditional CHD risk factors, including socioeconomic status. Further research is warranted in this important area of public health.

\section{Commentary}

The difficulty in assessing periodontal disease as a risk factor for to CHD has always been the result of standardised indices. Where one study relies almost exclusively on bone loss, another may only have the resources to examine tooth loss. Comparing one study with another directly may seem difficult, but if you look at the results of many similar studies, regardless of indices, each indicating a similar trend regardless of the disease marker chosen, the scientific community cannot help but give this tide of evidence some credence. Thus is the situation with proving an association between periodontal disease and CHD.

The evidence suggests that periodontal disease may be related to CHD by an oft theorised but as yet unproven pathway. Furthermore, research has reflected a belief that this association may be bidirec- tional, indicating that both disease processes may result from a common inflammatory pathway. This would seem to suggest that CHD, a disease with huge public health implications in morbidity and mortality, could be alleviated if the relationship could be more thoroughly extrapolated. Doing so could yield a treatment that was beneficial to not one but several ailments simultaneously.

This meta-analysis conducted by Humphrey and colleagues aims to explore the various investigations into the possible bidirectional causality between periodontal disease and CHD, and manages to do so despite the aforementioned lack of a standard index for either, having detailed those limitations accurately and fairly. Despite the discrepancy, the analysis has identified that a good deal of evidence exists that consistently reveals a statistically significant association between periodontal disease and CHD. A commendable feature that separates this study from others is the weighting they gave to the quality of evidence, rather than accepting results at face value.

Another aspect contained here is an attempt to explain the association from a biological standpoint, considering the possibility that a genetic inflammatory predisposition could account for the association, without ruling out the part that could be played by confounding factors such as smoking, diabetes and socioeconomic status, as well. The evidence has been approached from an unbiased and prospective view, and various alternative theories have been explained and argued fairly without being dismissed prematurely. Nonetheless, the analysis supports the view that periodontal disease is implicated in CHD with some degree of confidence. Such evaluation should continue in assessing the true bidirectional nature of these conditions, as most of the literature assessed seemed to focus on the causation of CHD without considering the possible dynamic between it and periodontal disease.

Aaron Cronin

Maxillofacial Unit, Sherwood Forest Hospitals NHS Trust, King's Mill Hospital, Sutton in Ashfield, Nottinghamshire, UK

Evidence-Based Dentistry (2009) 10, 22. doi:10.1038/sj.ebd.6400634 